\title{
Human LZIP induces monocyte CC chemokine receptor 2 expression leading to enhancement of monocyte chemoattractant protein 1/CCL2-induced cell migration
}

\author{
Ho Joong Sung ${ }^{1}$, Yoon Suk Kim ${ }^{2}$, Hyereen Kang ${ }^{1}$ \\ and Jesang $\mathrm{Ko}^{1,3}$ \\ ${ }^{1}$ School of Life Sciences and Biotechnology \\ Korea University \\ Seoul 136-701, Korea \\ ${ }^{2}$ Department of Biomedical Laboratory Science \\ College of Health Science, Yonsei University \\ Wonju 222-701, Korea \\ ${ }^{3}$ Corresponding author: Tel, 82-2-3290-3445; \\ Fax, 82-2-927-9028; E-mail, jesangko@korea.ac.kr \\ Accepted 13 March 2008
}

Abbreviations: CCR, CC chemokine receptor; MCP, monocyte chemoattractant protein; Lkn, leukotactin; MIP, macrophage inflammatory protein; C/EBP, CCAAT enhancer binding protein

\begin{abstract}
Chemokines and chemokine receptors play a role in migration of circulating leukocytes to the region of inflammation. Human LZIP is an uncharacterized transcription factor and is known to participate in leukotactin (Lkn)-1/CCL15-induced cell migration. We investigated the role of human LZIP in expression of CC chemokine receptors (CCRs) and its involvement in monocyte migration. RNase protection analysis showed that LZIP increased mRNA expression of CCR2 and CCR1 in THP-1 cells. Surface expressions of both CCR2 and CCR1 were also increased by LZIP. Results from an electrophoretic mobility shift assay showed that LZIP binds to the C/EBP element in the CCR2 promoter. LZIP also enhanced the chemotactic activities of monocyte chemoattractant protein-1/CCL2 and Lkn-1. These results suggest that LZIP regulates expression of chemokine receptors that are involved in monocyte migration.
\end{abstract}

Keywords: chemokines; chemotaxis; inflammation; monocytes; receptors, chemokine; transcription factors

\section{Introduction}

Chemokines are a superfamily of chemotactic cyto- kines that are categorized into four groups on the basis of their conservation of four cysteine residues and on their ability to induce leukocyte trafficking into sites of inflammation (Gerard and Rollins, 2001). The action of chemokines is mediated by their receptors, a family of seven transmembrane G-protein-coupled receptors (Gerard and Rollins, 2001). Evidence suggests that chemokines and their receptors are involved in development of inflammatory diseases including atherosclerosis (Nelken et al., 1991; Gu et al., 1998; Dawson et al., 1999; Reape and Groot, 1999; Yu et al., 2004; Ko et al., 2007).

CC chemokine monocyte chemoattractant protein (MCP)-1/CCL2 is a chemoattractant for monocytes. MCP-1 is induced by various stimuli, such as cytokines and oxidized adducts, and is secreted from endothelial cells, smooth muscle cells and macrophages (Myers et al., 1996). CC chemokine receptor (CCR) 2 is highly expressed in peripheral blood monocytes, macrophages, and $\mathrm{T}$ lymphocytes and it is the only established functional receptor for MCP-1 on hematopoietic cells (Boring et al., 1998; Berger et al., 1999; Han et al., 2000). Although MCP-1 and CCR2 are believed to play an important role in leukocyte trafficking and the development of atherosclerosis, little information is available regarding the mechanism of intracellular signaling events that regulates the expression of chemokine receptors.

The human leucine zipper protein LZIP is a ubiquitous transcription factor that belongs to the bZIP superfamily (Burbelo et al., 1994; Lu et al., 1997, 1998). LZIP is known to be involved in regulation of cell growth by binding to human host cell factor, which is involved in cell proliferation (Jin et al., 2000). It has also been suggested that LZIP serves a novel cellular tumor suppressor function that is targeted by the hepatitis C virus core (Jin et al., 2000). However, the exact biological role and the natural target genes of LZIP have not been characterized. Although there is controversy regarding the localization of endogenous LZIP, we have reported that LZIP physically interacts with CCR1 and participates in regulation of leukotactin (Lkn)-1/CCL15-induced cell migration without affecting the chemotactic activities of other CCR1-binding chemokines (Ko et al., 2004). We have also proposed that LZIP acts as a positive regulator in 
the $N F-\kappa B$ activation pathway that is triggered by Lkn-1, causing enhancement of Lkn-1-induced migration of monocytes (Jang et al., 2007a). Recently, it has been reported that the expression of LZIP is regulated by Lkn-1 (Jang et al., 2007b). These results indicate that LZIP probably functions as a cellular modulator as well as a transcription factor. Since LZIP participates in the development of inflammatory responses, we investigated whether LZIP plays a role in expression of CCR2, which is a major chemokine receptor involved in atherosclerosis.

\section{Materials and Methods}

\section{Materials}

Human THP-1 cells were purchased from American Type Culture Collection (ATCC). RPMI 1640, FBS and Trizol reagent were obtained from Invitrogen corp. (Gaithersburg, MD). RNase protection assay kit was obtained from BD bioscience (San Jose, CA). Recombinant human MCP-1, Lkn-1, macrophage inflammatory protein (MIP)-1 $\alpha / C C L 3$, and anti-CCR1 and anti-CCR2 antibodies were products of R\&D systems (Minneapolis, MN). FITC-conjugated goat anti-mouse $\lg G$ and rabbit polyclonal IgG-HA probe were purchased from Santa Cruz Biotechnology (Santa Cruz, CA). $\left[\alpha-{ }^{32}\right.$ P $]$ UTP and $\left[\gamma-{ }^{32}\right.$ P]ATP were from Amersham Pharmacia Biotech. (Uppsala, Sweden). Other ultra pure chemical reagents were purchased from Sigma-Aldrich (St. Louis, MO). Rabbit polyclonal anti-LZIP antibody was raised against an $\mathrm{N}$-terminal peptide (amino acids 2-29) conjugated to keyhole limpet hemocyanin.

\section{Cell culture and transient transfection}

THP-1 cells were grown in RPMI 1640 supplemented with $10 \%$ heat-inactivated FBS, penicillin (100 $\mathrm{U} / \mathrm{ml})$ and streptomycin $(100 \mu \mathrm{g} / \mathrm{ml})$. THP-1 cells were seeded into 6 -well plates at a density of $2 \times$ $10^{6}$ cells/well. Cells were transfected with $4 \mu \mathrm{g}$ of DNA using lipofectamine reagents (Invitrogen) according to the manufacture's instructions.

\section{FACS analysis}

Transfected cells were harvested and washed with PBS containing $0.5 \%$ BSA. Blocked cells with normal rabbit IgG were separated into new tubes. Each tube was added with PBS containing anti-HA, anti-CCR2, or anti-CCR1 antibodies. Baseline fluorescence was obtained from cells transfected with the mock vector alone. Following incubation and washings, cells were incubated for $30 \mathrm{~min}$ at $4^{\circ} \mathrm{C}$ with FITC-conjugated goat anti-mouse $\operatorname{lgG}$ or rabbit anti-rat IgG. Then cells were washed and analyzed on a FACSort cytofluorimeter (Becton Dickinson).

\section{Chemotaxis assay}

Migration of cells was monitored using a 48-well microchamber (Neuroprobe, Cabin John, MD) as previously described (Ko et al., 2002). Briefly, the lower wells were filled with $28 \mu \mathrm{l}$ buffer alone or with buffer containing MCP-1 or Lkn-1 and the upper wells were filled with $50 \mu$ of THP-1 cells at $1 \times 10^{6}$ cells $/ \mathrm{ml}$ in RPMI 1640 containing $1 \%$ BSA and $30 \mathrm{mM}$ HEPES. The two compartments were separated by a polyvinylpyrrolidone-free filter (Neuroprobe) with $5 \mu \mathrm{m}$ pores that was precoated with RPMI 1640 containing rat tail collagen type I at $4^{\circ} \mathrm{C}$ overnight. After incubation for $6 \mathrm{~h}$ at $37^{\circ} \mathrm{C}$, the filters were removed from the chamber, washed, fixed, and stained with Diff-Quick (Baxter, Deerfield, IL). The cells of two randomly selected oilimmersed fields were counted using Axiovert 25 (Carl Zeiss, Jena, Germany) and Visus Image Analysis System (Foresthill Products, Foresthill, $\mathrm{CA})$. The chemotactic index (CI) was calculated from the number of cells that migrated to the control.

\section{RNase protection assay}

THP-1 cells were seeded into 6 -well plates at $2 \times$ $10^{6}$ cells/well and cultured in RPMI 1640. After transfection, the cells were harvested and total RNA was extracted using Trizol reagent (Invitrogen) as described by manufacturer's instruction. The hCR5 template set containing DNA templates for CCR1, CCR3, CCR4, CCR5, CCR8, CCR2a+b, L32, and GAPDH was purchased from BD bioscience. $\left[\alpha-{ }^{32}\right.$ P]UTP-labeled RNA probes were synthesized from the hCR5 template set by T7 RNA polymerase. Probes were hybridized overnight with target RNA. Free probes and other single-stranded RNA were digested with RNase, followed by proteinase $\mathrm{K}$ treatment and phenol chloroform extraction. After ethanol precipitation with $4 \mathrm{M}$ ammonium acetate, the RNase-protected probes were dissolved in loading buffer. Denaturing polyacrylamide gel $(4.75 \%)$ was pre-electrophoresed in 0.5 $\times$ Tris-borate EDTA (TBE) buffer for $30 \mathrm{~min}$ prior to loading and electrophoresis continued at $55 \mathrm{~W}$ for $2 \mathrm{~h}$. Dried gel was visualized by autoradiography. Specific bands were identified by their individual migration patterns in comparison with the undigested probes. The bands were normalized 
with GAPDH band.

\section{Electrophoretic mobility shift assay (EMSA)}

Nuclear extracts were prepared from confluent flasks of the mock vector or HA-LZIP transfected THP-1 cells. The oligonucleotides of CCAAT/enhancer binding proteins (C/EBP) binding site in CCR2 promoter $(-324 \sim-298)$ were synthesized. The oligonucleotide was 5 '-end-labeled with $\left[\gamma^{-32}\right.$ P]ATP using T4 polynucleotide kinase (Promega, Madison, WI). Unincorporated nucleotide was removed by passage over a Bio-Gel P-6 spin column (Bio-Rad, Hercules, CA). Nuclear extracts (15 $\mu \mathrm{g}$ of total protein) were incubated with radiolabeled probe for $20 \mathrm{~min}$ at room temperature, and protein-DNA complexes were separated from free probe by electrophoresis on a $4 \%$ native polyacrylamide gel in $0.5 \times$ Tris- $\mathrm{HCl}(\mathrm{pH}$ 7.5), $1 \mathrm{mM} \mathrm{MgCl}_{2}, 50 \mathrm{mM} \mathrm{NaCl}, 0.5 \mathrm{mM}$ EDTA, $4 \%$ glycerol, $0.5 \mathrm{mM}$ DTT, and $50 \mu \mathrm{g} / \mathrm{ml}$ of poly $(\mathrm{dl}-\mathrm{dC}) \cdot \operatorname{poly}(\mathrm{dl}-\mathrm{dC})$. Gels were pre-electrophoresed in $0.5 \times$ TBE for 30 min prior to loading and electrophoresis continued for approximately $3 \mathrm{~h}$ until the bromophenol blue dye approached the bottom of the gel. Dried gels were visualized by autoradiography. For competition experiments, binding reactions were incubated with 20 -fold molar excess of unlabeled C/EBP binding oligonucleotide for $20 \mathrm{~min}$ before addition of the radiolabeled oligonucleotide.

\section{Results}

\section{LZIP increases mRNA expressions of CCR2 and CCR1 in THP-1 cells}

To investigate the effect of LZIP on mRNA expression of selected CC chemokine receptors, we performed an RNase protection assay in THP-1 cells transfected with the mock vector or LZIP. CCR2 and CCR 1 mRNA expressions were increased in cells transfected with LZIP compared to cells transfected with the mock vector (Figure 1A). CCR3 and CCR4 mRNA expressions were slightly increased by LZIP (Figure 1A). Yeast tRNA was used as a negative control and GAPDH was used for normalization of samples. Figure 1B shows the transfection efficiency of THP-1 cells analyzed using a FACSorter cytofluorimeter. These results indicate that LZIP up-regulates mRNA expression of chemokine receptors, including CCR2 and CCR1.

\section{Surface expressions of CCR2 and CCR1 are increased by LZIP}

Since LZIP increased mRNA expressions of CCR2
A

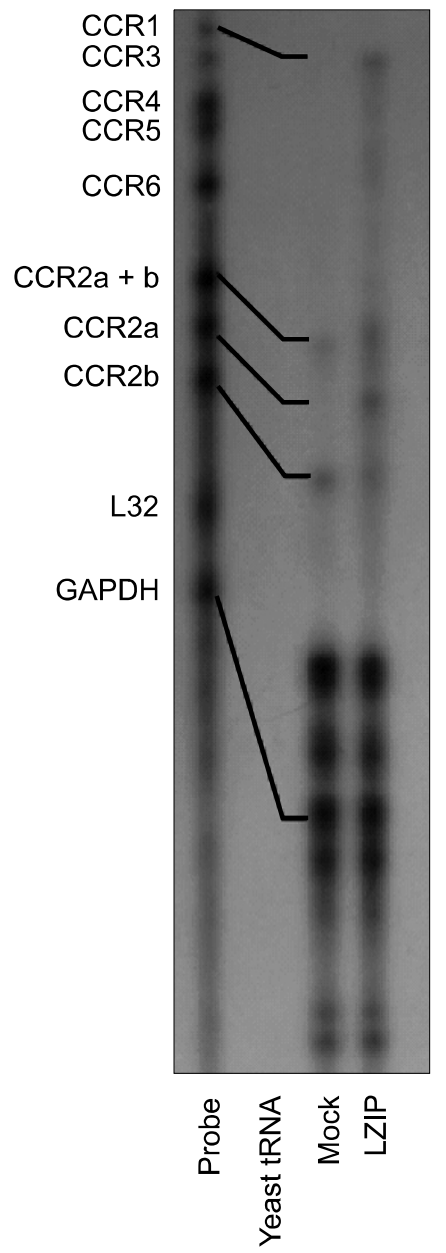

B

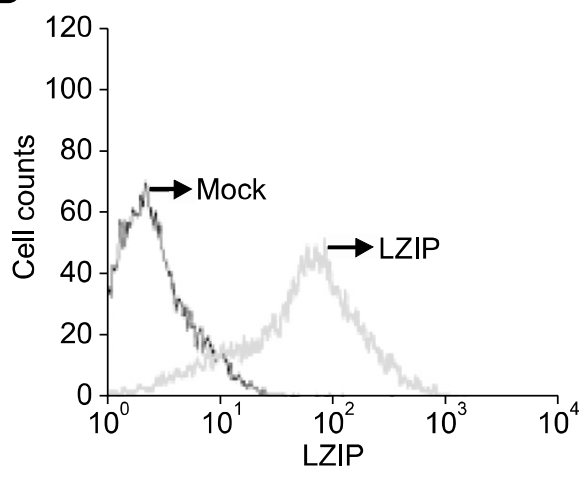

Figure 1. LZIP increases mRNA expressions of CCR2 and CCR1 in THP-1 cells. (A) THP-1 cells were transfected with the mock vector or HA-LZIP and total RNA was extracted from transfected cells. The mRNA levels of CCRs were analyzed by RNase protection assay using template sets as described in methods. Specific bands were detected by comparing with the undigested probes. The bands were normalized with GAPDH and L32 bands. Data are expressed as representative of three individual experiments. (B) Expression of transfected LZIP was confirmed by fluorescence-activated cells sorter using polyclonal HA probe. 
A

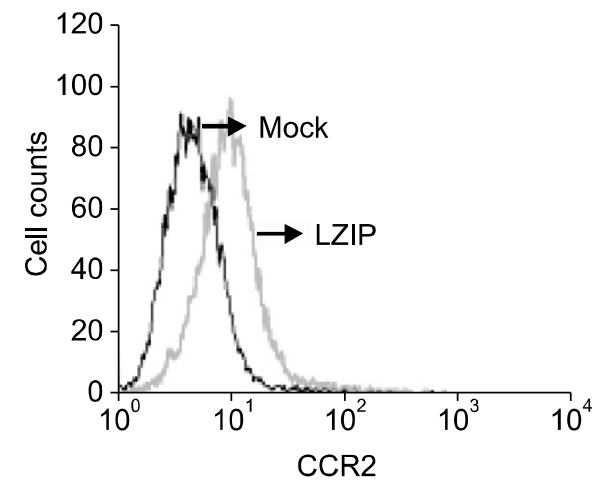

B

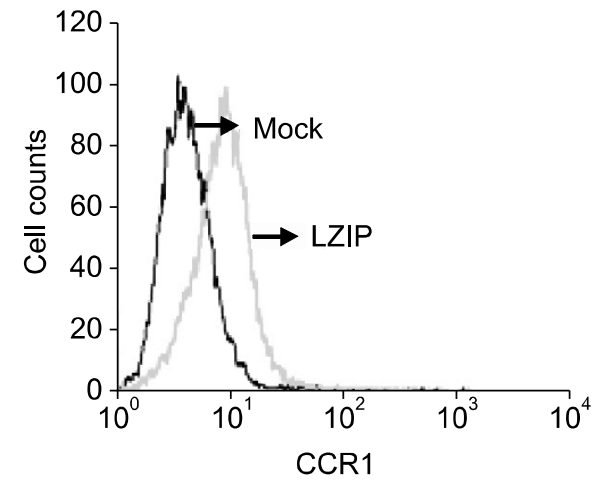

Figure 2. Surface expressions of CCR2 and CCR1 are increased by LZIP. THP-1 cells were transfected with the mock vector or HA-LZIP. Surface expressions of CCR2 (A) and CCR1 (B) were analyzed by fluorescence-activated cells sorter using monoclonal anti-CCR2 and anti-CCR1 antibodies. Data are expressed as representative of three individual experiments. and CCR1, we examined whether surface protein expressions of CCR2 and CCR1 are affected by LZIP. Strong CCR2 and CCR1 surface expressions were detected by flow cytometry in LZIP transfected THP-1 cells compared to cells transfected with the mock vector (Figure 2). CCR2 and CCR1 surface expressions were increased by LZIP 1.9- and 2.1 -fold, respectively. These results correlate with CCR2 and CCR1 mRNA expressions due to LZIP. These data indicate that LZIP up-regulates mRNA and protein expressions of both CCR2 and CCR1.

\section{LZIP binds to the C/EBP element in CCR2 promoter}

CCR2 promoter contains the CCAAT enhancer binding protein (C/EBP) element (Yamato et al., 1999), and LZIP binds to the C/EBP element and activates transcription from C/EBP-containing reporter genes (Lu et al., 1997). To investigate the mechanism of CCR2 mRNA expression, we performed an electrophoretic mobility shift assay (EMSA) using a ${ }^{32} \mathrm{P}$-labeled C/EBP element as a probe. Figure $3 \mathrm{~A}$ shows a shifted LZIP nuclear complex bound to the probe. Cells transfected with LZIP showed an increase in the formation of this complex. To determine the specificity of LZIP binding activity, nuclear extracts were incubated with the labeled LZIP binding probes in the absence and presence of a 20 -fold molar excess of unlabeled LZIP binding probes. Competition analysis showed that the LZIP binding complex competed with the unlabeled LZIP binding probes (Figure 3B), indicating that the LZIP binding activity is specific. These data indicate that LZIP binds to the C/EBP element and induces CCR2 mRNA and protein expression.

\section{LZIP enhances THP-1 cell migration in response to MCP-1 and Lkn-1}

Cell migration is the primary cellular event initiated
A

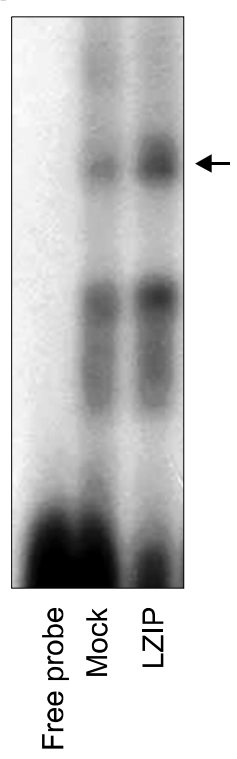

B

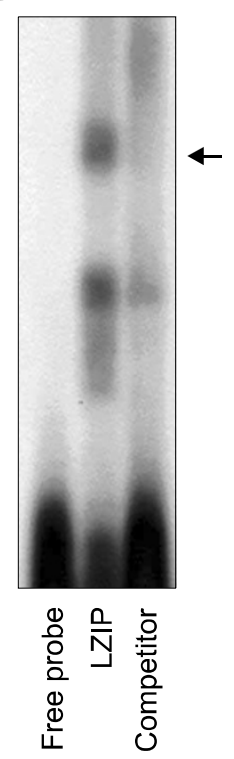

Figure 3. LZIP binds to the C/EBP element in CCR2 promoter. Nuclear extracts $(15 \mu \mathrm{g})$ from mock or HA-LZIP transfected THP-1 cells were prepared and subjected to EMSA for the DNA binding activity of LZIP with ${ }^{32} \mathrm{P}$-labeled oligonucleotides in the absence $(\mathrm{A})$ and presence $(\mathrm{B})$ of 20 -fold molar excess of unlabeled competitors.

by the chemokine receptor interacting with chemokine. Therefore, we investigated whether LZIP affects the chemotactic activities of MCP-1 and Lkn-1 in THP-1 cells. To examine the chemotactic activity in LZIP transfected THP-1 cells, a cell migration assay was performed in a 48 well microchamber. MCP-1 showed the typical bell-shape curve in THP-1 chemoattraction with the peak of the curve at $10 \mathrm{ng} / \mathrm{ml}$ (Figure 4A) and Lkn-1 showed a maximum activity at $100 \mathrm{ng} / \mathrm{ml}$ (data not shown). THP-1 cell migration in response to MCP-1 was increased by 2-fold based on the chemotactic index in cells transfected with LZIP (Figure 4A). 
A

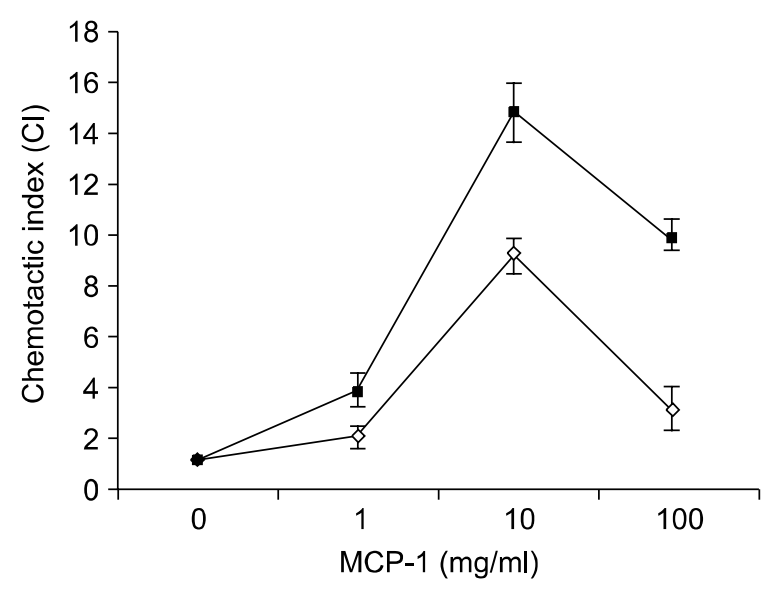

B

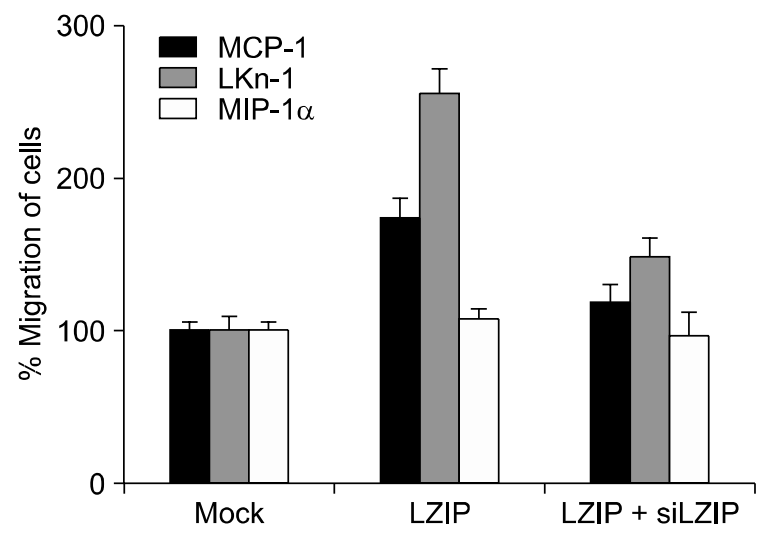

Figure 4. LZIP enhances THP-1 cell migration in response to MCP-1 and Lkn-1. (A) THP-1 cells transfected with the mock vector $(\diamond)$ or HA-LZIP $(\square)$ were incubated with the indicated concentrations of MCP-1. (B) THP-1 cells transfected with the mock vector, HA-LZIP or siLZIP were incubated with MCP-1 $(10 \mathrm{ng} / \mathrm{ml})$, Lkn-1 $(100 \mathrm{ng} / \mathrm{ml})$, and MIP-1 $\alpha(1 \mathrm{ng} / \mathrm{ml})$. After incubation for $5 \mathrm{~h}$, filters were stained with Diff-Quick. The number of cells that migrated was counted microscopically in four randomly selected fields per well. The chemotactic index $(\mathrm{Cl})$ was calculated from the number of cells migrating to the test chemokines divided by that migrating to the controls. Data are expressed as mean $\mathrm{Cl} \pm \mathrm{SEM}$ of three independent experiments.

The chemotactic activity of Lkn-1 was increased by 2.7 -fold at $100 \mathrm{ng} / \mathrm{ml}$ in LZIP transfected cells compared with cells transfected with the mock vector (Figure 4B). However, the chemotactic activity of MIP-1 $\alpha$, used as a negative control in LZIP transfected cells, was comparable with cells transfected with the mock vector (Figure 4B). We generated siRNA for LZIP (siLZIP) that can reduce the expression of LZIP to $0-50 \%$ of the endogenous level (Ko et al., 2004). Transfection experiments with LZIP and siLZIP reduced the chemotactic activities of MCP-1 and Lkn-1 in cells transfected with LZIP alone by $37 \%$ and $40 \%$, respectively (Figure 4B). These data indicate that LZIP upregulates CCR2 and CCR 1 expressions at both the mRNA and protein levels in THP-1 cells and enhances the chemotactic activities of MCP-1 and Lkn-1.

\section{Discussion}

CCR2 and MCP-1 are known to be important molecules in the development of atherosclerosis. During the early stage of atherogenesis, CCR2 and MCP-1 play important roles in atherosclerotic plaque formation via accumulation of circulating monocytes in the arterial wall. We investigated the role of uncharacterized transcription factor LZIP in expression of CC chemokine receptors. We demonstrated that, 1) LZIP increases mRNA expressions of CCR2 and CCR1 in THP-1 cells, 2) surface expressions of CCR2 and CCR 1 are increased by
LZIP, 3) LZIP binds to the C/EBP element in CCR2 promoter and 4) LZIP enhances THP-1 cell migration in response to MCP-1 and Lkn-1.

Results from RPA analysis indicated that LZIP increases CCR2 mRNA expression. CCR2 mRNA expression in THP-1 cells transfected with the mock vector was very low, however, cells transfected with LZIP showed an increase in CCR2 mRNA expression. LZIP also increased CCR1 mRNA expression. Our previous report suggests that LZIP functions as a cellular modulator of CCR1dependent chemokines and is involved in Lkn1-induced cell migration (Ko et al., 2004). CCR1 mRNA expression in THP-1 cells transfected with the mock vector was very low, however, cells transfected with LZIP showed an increase in CCR1 mRNA expression. Surface expressions of CCR2 and CCR 1 were also increased by LZIP, indicating that LZIP plays a role in the expressions of CCR2 and CCR1 both at the mRNA and at the protein level, and probably functions as a positive regulator of CCR2 and CCR1 involved in inflammatory responses.

To determine the regulatory mechanism of LZIP in CCR2 expression, we analyzed the promoter region of CCR2. LZIP is a basic leucine zipper transcription factor that binds consensus DNA elements, such as CRE, AP-1 and C/EBP (DenBoer et al., 2005). Based on reports regarding the nucleotide sequence of the human CCR2 gene (Yamato et al., 1999), C/EBP elements exist in CCR2 promoter. Results from EMSA show that LZIP binds to the C/EBP element in CCR2 pro- 
moter, indicating that LZIP functions as a transcription factor that binds to the C/EBP element of CCR2 and accelerates the transcriptional activation of CCR2. Although further studies are required to characterize the exact regulatory mechanism of LZIP in expression of chemokine receptors, our data are consistent with previous results that LZIP binds C/EBP element (DenBoer et al., 2005).

Transmigration of monocytes through endothelial monolayers is induced by chemotaxis, which is an essential step for complete monocyte recruitment to the vessel wall. We showed that LZIP modulates MCP-1-mediated monocyte chemotactic activity and Lkn-1-mediated chemotaxis. MCP-1 and Lkn-1 are known chemokines that are involved in atherosclerosis (Gerard and Rollins, 2001; Yu et al., 2004). Since LZIP did not affect the chemotactic activity of MIP-1 $\alpha$, LZIP possibly participates in the development of atherosclerosis via an increase in CCR2 and CCR1 expressions and subsequent induction of the chemotactic activities of MCP-1 and Lkn-1.

Herein, we report important roles for LZIP in expression of chemokine receptors and subsequent enhancement of monocyte migration. Our results indicate that LZIP regulates the expressions of the chemokine receptors CCR2 and CCR1 that are involved in the early stages of atherogenesis. The modulation effect of CCR2 expression is due to the DNA binding ability of LZIP to the C/EBP element in CCR2 promoter. In conclusion, we characterized the function of LZIP in regulation of the chemokine receptors that are involved in atherosclerosis. Further characterization of the role of LZIP in atherogenesis will contribute to an understanding of the development of atherosclerosis and the regulatory mechanisms of the chemokines and chemokine receptors that are involved in atherosclerosis.

\section{Acknowledgment}

This work was supported by a grant of the Korea Health 21 R\&D Project, Ministry of Health and Welfare, Republic of Korea (A06-0605-AA1018-07A2-00010A) and a grant from the Diseases Network Research Program (M10751050002-07N5105-00210), Ministry of Science and Technology, South Korea.

\section{References}

Berger O, Gan X, Gujuluva C, Burns AR, Sulur G, Stins M, Way D, Witte M, Weinand M, Said J, Kim KS, Taub D, Graves MC, Fiala M. CXC and CC chemokine receptors on coronary and brain endothelia. Mol Med 1999;5:795-805
Boring L, Gosling J, Cleary M, Charo IF. Decreased lesion formation in CCR2-/- mice reveals a role for chemokines in the initiation of atherosclerosis. Nature 1998;394:894-7

Burbelo PD, Gabriel GC, Kibbey MC, Yamada Y, Kleiman HK, Weeks BS. LZIP-1 and LZIP-2: two novel members of the bZIP family. Gene 1994;139:241-5

Dawson TC, Kuziel WA, Osahar TA, Maeda N. Absence of CC chemokine receptor-2 reduces atherosclerosis in apolipoprotein E-deficient mice. Atherosclerosis 1999;143: 205-11

DenBoer LM, Hardy-Smith PW, Hogan MR, Cockram GP, Audas TE, Lu R. Luman is capable of binding and activating transcription from the unfolded protein response element. Biochem Biophys Res Commun 2005;27:113-9

Gerard G, Rollins BJ. Chemokines and disease. Nat Immunol 2001;2:108-15

Gu L, Okada Y, Clinton SK, Gerard C, Sukhova GK, Libby P, Rollins BJ. Absence of monocyte chemoattractant protein-1 reduces atherosclerosis in low density lipoprotein receptordeficient mice. Mol Cell 1998;2:275-81

Han KH, Chang MK, Boullier A, Green SR, Li A, Glass CK, Quehenberger O. Oxidized LDL reduces monocyte CCR2 expression through pathways involving peroxisome proliferatoractivated receptor $\gamma$. J Clin Invest 2000;106:793-802

Jang SW, Kim YS, Kim YR, Sung HJ, Ko J. Regulation of human LZIP expression by NF-אB and its involvement in monocyte cell migration induced by Lkn-1. J Biol Chem 2007a;282:11092-100

Jang SW, Kim YS, Lee YH, Ko J. The role of human LZIP in differential activation of the NF- $\kappa B$ pathway that is induced by CCR1-dependent chemokines. J Cell Physiol 2007b; 211:630-7

Jin DY, Wang HL, Zhou Y, Chun AC, Kibler KV, Hou YD, Kung $\mathrm{H}$, Jeang $K T$. Hepatitis $C$ virus core protein-induced loss of LZIP function correlates with cellular transformation. EMBO J 2000;19:729-40

Ko J, Kim IS, Jang S-W, Lee YH, Shin SY, Min DS, Na DS. Leukotactin-1/CCL15-induced chemotaxis signaling through CCR1 in HOS cells. FEBS Lett 2002;515:159-64

Ko J, Jang S-W, Kim YS, Kim IS, Sung HJ, Kim H-H, Park J-Y, Lee YH, Kim J, Na DS. Human LZIP binds to CCR1 and differentially affects the chemotactic activities of CCR1dependent chemokines. FASEB J 2004;18:890-2

Ko J, Yun C-Y, Lee J-S, Kim J-H, Kim IS. p38 MAPK and ERK activation by 9 -cis-retinoic acid induces chemokine receptors CCR1 and CCR2 expression in human monocytic THP-1 cells. Exp Mol Med 2007;39:129-38

Lu R, Yang P, O'Hare P, Misra V. Luman, a new member of the CREB/ATF family, binds to herpes simplex virus VP16-associated host cellular factor. Mol Cell Biol 1997;17: 5117-26

Lu R, Yang P, Padmakumar S, Misra V. The herpesvirus transactivator VP16 mimics a human basic domain leucine zipper protein, luman, in its interaction with HCF. J Virol 1998; 72:6291-7 
Myers SJ, Wong LM, Charo IF. Signal transduction and ligand specificity of the human monocyte chemoattractant protein-1 receptor in transfected embryonic kidney cells. J Biol Chem 1996;270:5786-92

Nelken NA, Coughlin SR, Gordon D, Wilcox JN. Monocyte chemoattractant protein-1 in human atheromatous plaques. J Clin Invest 1991;88:1121-7

Reape TJ, Groot PH. Chemokines and atherosclerosis. Atherosclerosis 1999;147:213-25
Yamato K, Takeshima H, Harnada K, Nakao M, Kino T, Nishi T, Kochi M, Kuratsu J, Yoshimura T, Ushio Y. Cloning and functional characterization of the 5'-flanking region of the human monocyte chemoattractant protein-1 receptor (CCR2) gene. Essential role of 5'-untranslated region in tissue-specific expression. J Biol Chem 1999;274:4646-54

Yu R, Kim CS, Kawada T, Kwon TW, Lim TH, Kim YW, Kwon BS. Involvement of leukotactin-1, a novel CC chemokine, in human atherosclerosis. Atherosclerosis 2004;174:35-42 